\title{
The Kadomtsev-Petviashvili Equations and Fundamental String Theory *
}

\author{
Gerald Gilbert * \\ California Institute of Technology, Pasadena, CA 91125, USA
}

\begin{abstract}
In this paper the infinite sequence of non-linear partial differential equations known as the Kadomtsev-Petviashvili equations is described in simple terms and possible applications to a fundamental description of interacting strings are addressed. Lines of research likely to prove useful in formulating a description of non-perturbative string configurations are indicated.
\end{abstract}

\section{Introduction}

The state of current understanding of string physics is incomplete and far from satisfactory. The theory of the dynamical evolution of entire strings as such, consistent with and following from the principles of quantum mechanics, has yet to be elucidated. Conventional wisdom would hold that, viewed as a system in quantum mechanics, an assemblage of strings ought to be describable by a Hamiltonian. For now, however, the true Hamiltonian of heterotic strings subject to mutual interaction remains undiscovered. Also undiscovered is the manifestly invariant path-integral formulation of such a system. Alternatively one might flaunt conventional wisdom and hope that the quantum physics of interacting heterotic strings could be deduced without ever "writing down a Lagrangian and quantizing." After all there exist in (perhaps not coincidentally) two dimensions a number of conformal quantum field theories characterized by an infinite number of conserved quantities for which no Lagrangian is known. Of course in these cases one knows what to do; for example the quantum field theory may be formulated entirely in terms of Ward identities [1]. For string theory in the absence of a Hamiltonian, in contrast, it is at present entirely unclear as to how to proceed in describing the quantum theory to allow for the computation of, for instance, nonperturbative effects. Indeed it is not at all obvious how some computations usually

\footnotetext{
* Work supported in part by U.S. Department of Energy under Contract No. DEAC-03-81-ER40050

$\star \star$ Weingart Research Fellow in Theoretical Physics
} 
regarded as "perturbative" can be performed in lieu of a Hamiltonian either. The foremost example is the problem of understanding a collection of strings interacting in canonical ensemble at extremely high temperatures in the very early Universe.

Since an underlying heterotic string Hamiltonian is not known, we cannot at present compute the trace of $\exp (-\beta \hat{H})$ and thus we cannot directly investigate string thermodynamics. (In traditional finite-temperature field theory we deduce the proper periodicity for the quantum fields as well as the proper quantization of the time-components of four-vectors only after evaluating Tr $e^{-\beta \hat{H}}$ first. It would seem that existing papers devoted to the study of strings at finite temperature employ choices of thermodynamic boundary conditions which must be regarded for now as well-intentioned guesses.)

Actually, it is the case that there is really no true quantum theory of strings extant at present. Instead the totality of string theory today consists of some disconnected pieces without a central logical foundation. A coherent formulation of interacting strings has been sought for the last few years in the form of a stringtheoretic extension of quantum (point-)field theory [2-6]. This approach has not succeeded for the heterotic string so far and may not be successful ultimately, and we will throughout refer to the objective of our search, therefore, as fundamental string theory.

Apart from the need for a fundamental string theory as a matter of principle and our interest in studying non-perturbative configurations, we are anxious to solve a number of long-standing problems of the first-quantized approach. Up to the present, research on the calculation of the perturbative approximation to (firstquantized) quantum mechanical amplitudes for closed, oriented strings has yielded a fairly clear but impracticable method [7-9]. (The clarity is somewhat greater for the closed bosonic string than for the heterotic string owing to the absence of supermoduli for the former; this virtue is offset by the fact that the bosonic amplitudes don't exist.) The picture as it has developed allows one to calculate terms in the perturbative expansion in basically two ways [10-13]. If one chooses a real parametrization of the Teichmüller space of conformal equivalence classes of metrics on the world-sheet, then one must evaluate an integral the integrand of which is a function of Selberg zeta-functions. Alternatively one may choose to take advantage of the fact that there is a complex structure associated with the moduli space for a compact Riemann surface. Then one must evaluate an integral the integrand of which is a function of Riemann theta-functions.

In the former case the integral cannot be exactly evaluated when the worldsurface has at least two handles for two reasons. First, the Selberg zeta-function depends implicitly on the real moduli through the lengths of representatives of primitive conjugacy classes of the Fuchsian group of the surface. However, it appears almost impossible to express the primitive lengths explicitly and usefully in terms of the moduli. Second, the integral is to be evaluated over the fundamental domain of the mapping class group, the "boundaries" of which (viewed as a subspace in the Teichmüller space) are unknown for surfaces more complicated than the torus.

In the latter case the integral can generally not be exactly evaluated as well. The Riemann theta-function depends on the complex moduli through the elements of 
the period matrix of the Riemann surface. Unfortunately the computationally useful "similarity" between the space of moduli (with complex dimension $3 g-3$ where $g$ is the genus) and a fundamental domain in the Siegel upper half-plane [14] [with complex dimension $\left.\frac{1}{2} g(g+1)\right]$ describing inequivalent period matrices stops abruptly at $g=4$.

It is true that by examining the integrals over degenerating Riemann surfaces we can extract certain interesting and useful limiting values [15]. Nevertheless we are not able to directly evaluate almost all of the terms in the perturbative expansion of these amplitudes.

It has been suggested that in order to make progress it will be necessary to develop a method of describing string configurations which treats world-surface of different topology (including degenerating and degenerate world-surfaces) in a uniform manner [16]. Furthermore, in order to study non-perturbative configurations it seems plausible that Riemann surfaces of "infinite genus" will play an important role $[17,18]$. Recently it has been pointed out in the physics literature that infinite-dimensional Grassmannian manifolds may serve as the proper framework for such a description. One approach to infinite Grassmannians is as the space of choices of fermion boundary conditions for the free fermion field theory on a disc. This approach has been expounded by Segal and Wilson [19], and more recently by Witten [20]. A different approach is to describe the infinite Grassmannian in terms of the space of solutions of an infinite sequence of nonlinear partial differential equations known as the Kadomtsev-Petviashvili (KP) equations [21-23].

In this paper the KP equations are described in a simple and straightforward way. This unending sequence of exactly solvable non-linear equations is extraordinarily interesting in its own right.

The solutions of these equations are solitons, i.e., waves which scatter elastically with speed directly proportional to the amplitude. A number of other known sequences of exactly-solvable non-linear partial differential equations can be shown to reduce to special cases of the K P equations. The original KadomtsevPetviashvili equation (the first element of the KP sequence of equations) was discovered in 1970 in an effort to understand the propagation of long, shallow waves in plasma [24]. The rest of the sequence (gotten from the first equation in a systematic way as described in the sequel) was quickly discovered and general solutions worked out.

For applications to string physics there are at least three remarkable features of interest regarding the KP equations. First, the solutions of the entire sequence essentially furnish a representation of the Virasoro algebra [25]. Since the true raison d'etre of the Virasoro algebra in string theory is not fully understood at present, it is highly desirable to study new representations to gain a better perspective. Second, the solutions of the KP equations correspond to particular points in the infinite Grassmannian which in turn correspond to second-quantized Hilbert space state vectors for a free fermion field theory prepared on the boundary of the disc $[19,26]$. As the KP equations are exactly solvable, we can therefore obtain explicit expressions for these second-quantized Hilbert space vectors. Third, the KP sequence begs to be examined in the infinite limit. In this limit one analyzes an equation in an infinite number of independent variables. We will see 
later that these independent variables are in one-to-one correspondence with the oscillator modes of the string; thus in this limit we are considering all the string modes. Now, we will also see that different solutions of each equation in the KP sequence can be found corresponding to different values of the genus of a closed Riemann surface. Hence the KP sequence in the infinite limit is tailor-made to aid the precise study of "infinite-genus" Riemann surfaces for a possible concrete description of non-perturbative string configurations. Very little has been worked out formally regarding infinite-genus Riemann surfaces [27, 28]. Although a great deal more mathematical work needs to be done in this area before our understanding of these surfaces approaches in depth that of finite-genus surfaces, the "KP-approach" seems to be very promising.

In this paper an effort has been made to avoid an overly abstract and mathematical approach to this subject. Further work in this area is strongly motivated by indications that we will be rewarded with insight into a formulation of fundamental string theory. It is likely that much more work, a substantial fraction of which must be pure mathematical, will be carried out before a concrete formulation can be written down.

\section{The Kadomtsev-Petviashvili Equations}

We will need to employ elements of the theory of Riemann surfaces, functions of several complex variables and a little algebraic geometry in order to introduce and solve the KP equations. Here we will assert the needed elements in a non-rigorous way and refer the reader to any standard textbook on Riemann surfaces and thetafunctions for a fuller treatment.

We will be concerned primarily with compact, oriented Riemann surfaces with a finite number of handles in most of this paper. On such a manifold, differential one-forms which are holomorphic are said to be abelian of the first kind. It is a theorem that for a compact surface of genus $g$ there are precisely $g$ linearly independent abelian differentials of the first kind. If position on the surface is parametrized by a local coordinate $z$, then a holomorphic one-form $\omega(z)$ is defined by

$$
\omega(z)=f(z) d z,
$$

where $f(z)$ is an analytic function of its argument, and thus

$$
\bar{\partial} f=0
$$

so we see that holomorphic one-forms are necessarily closed (but not exact).

Associated with each of the $g$ independent abelian differentials of the first kind are a pair of contour integrals. These $2 g$ integrals, the periods of the differentials, are evaluated about $2 g$ closed contours which serve as the (arbitrarily chosen) basis for the (first) homology group $H_{1}$ of the Riemann surface. (For a compact $n$-dimensional manifold there are $n+1$ types of homology $H_{0}, \ldots, H_{n}$; for now we will consider only the first homology group.) For our immediate purposes the role of the first homology group is that the generators of that group, which we choose as the $2 g$ contours $a_{i}, b_{i}(i=1, \ldots, g)$ indicated in Fig. 1, "construct" all possible 


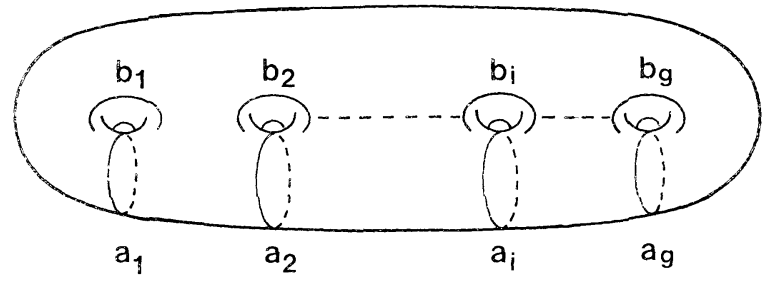

Fig. 1. This figure depicts a choice of basis for homology on a compact Riemann surface

closed contours on the Riemann surface. This means that for any meromorphic function $h(z)$ we may always write

$$
\oint_{C} h(z) d z=\sum_{i=1}^{g}\left[m_{i} \oint_{a_{2}} h(z) d z+n_{i} \oint_{b_{i}} h(z) d z\right]
$$

where the $m_{i}$ and the $n_{i}$ are collections of integers and $C$ is an arbitrary closed contour which is chosen to avoid the poles of $h(z)$.

We separate the $2 g$ homology basis contours (cycles) into two non-intersecting types $\mathbf{a}$ and $\mathbf{b}$; then the periods of the holomorphic differentials associated with one set (we choose a) can be represented as a matrix $M$. If we denote the holomorphic differentials by $v_{i}$ (assumed non-zero), then

$$
\oint_{a_{\imath}} v_{j} \equiv M_{i j}
$$

and we will have $\operatorname{det} M_{i j} \neq 0$, since otherwise there would exist a non-trivial differential form $v \equiv \sum_{i=1}^{g} \kappa_{i} v_{i}\left(\kappa_{i}\right.$ a set of constants) with zero a-periods. However this would contradict an easy theorem which states that if all the $a$-periods of a holomorphic differential vanish then the form itself vanishes identically. In virtue of the non-vanishing of the determinant of $M$ we may define a new set of $g$ linearly independent holomorphic differentials

$$
\omega_{i} \equiv \sum_{j=1}^{g} M_{i j}^{-1} v_{j}
$$

so that now

$$
\oint_{a_{2}} \omega_{j}=\delta_{i j} .
$$

The $g$ by $g$ matrix of $b$-periods is then defined to be the period matrix of the surface, denoted by $\Omega$ :

$$
\oint_{b_{1}} \omega_{j} \equiv \Omega_{i j}
$$

We know that $\boldsymbol{\omega}=\left(\omega_{1}, \ldots, \omega_{g}\right)$ is holomorphic and therefore closed; thus we have $\omega \wedge \omega^{\prime}=0$ and so

$$
0=\sum_{k=1}^{g}\left(\oint_{a_{k}} \omega_{i} \oint_{b_{k}} \omega_{j}-\oint_{b_{k}} \omega_{i} \oint_{a_{k}} \omega_{j}\right),
$$

which implies $\Omega_{i j}=\Omega_{j i}$. It is equally easy to show that $\operatorname{Im} \Omega>0$. 
We now introduce the standard Riemann theta-functions as generalizations to genus $g$ of the familiar Jacobi theta-functions of the torus. We write the $g$-fold infinite series

$$
\begin{aligned}
& \sum_{N_{1}=-x}^{\infty} \ldots \sum_{N_{g}=-\infty}^{\infty} \exp \left\{2 \pi i \left[\frac{1}{2}\left(N_{1}+\frac{a_{1}}{2}, \ldots, N_{g}+\frac{a_{g}}{2}\right)\left(\begin{array}{ccc}
\Omega_{11} & \ldots & \Omega_{1 g} \\
\vdots & \ddots & \vdots \\
\Omega_{g 1} & \ldots & \Omega_{g g}
\end{array}\right)\right.\right. \\
& \left.\left.\times\left(\begin{array}{c}
N_{1}+\frac{a_{1}}{2} \\
\vdots \\
N_{g}+\frac{a_{g}}{2}
\end{array}\right)+\left(N_{1}+\frac{a_{1}}{2}, \ldots, N_{g}+\frac{a_{g}}{2}\right)\left(\begin{array}{c}
z_{1}+\frac{b_{1}}{2} \\
\vdots \\
z_{g}+\frac{b_{g}}{2}
\end{array}\right)\right]\right\} \theta\left[\begin{array}{l}
\mathbf{a} \\
\mathbf{b}
\end{array}\right](z \mid \Omega),
\end{aligned}
$$

which defines the theta-function (in particular of a Riemann surface). Here the theta-character $\left[\begin{array}{l}\mathbf{a} \\ \mathbf{b}\end{array}\right]$ is composed of zeroes and integers and is called even or odd according as $\mathbf{a} \cdot \mathbf{b}=0$ or 1 (modulo 2). It is clear that there are $2^{2 q}$ distinct choices possible for the theta-characters. We have $\mathbf{z}=\left(z_{1}, \ldots, z_{g}\right) \in \mathbb{C}^{g}$ (i.e., not $\left.\mathbb{C}^{g} \cup \infty^{g}\right)$. $\Omega$ is a matrix with positive-definite imaginary part. We may take $\Omega$ to be symmetric since an antisymmetric part contributes nothing to the sum. Such a matrix is called a Riemann matrix; for now we will assume $\Omega$ is actually a period matrix. The series in Eq. (9) converges both uniformly and absolutely and has a host of interesting properties. In particular the theta-function is quasi-periodic, i.e.,

where

$$
\theta(\mathbf{z}+\mathbf{m}+\boldsymbol{\Xi})=\exp \left[-\frac{\pi i}{2} \mathbf{n} \cdot(\boldsymbol{\Xi}+2 \mathbf{z})\right] \theta(\mathbf{z}),
$$

$$
\theta\left[\begin{array}{l}
0 \\
0
\end{array}\right](\mathbf{z} \mid \Omega) \equiv \theta(\mathbf{z}),
$$

$\mathbf{m}$ and $\mathbf{n}$ are vectors of integers, and

$$
\Xi \equiv \sum_{j=1}^{g} n_{j} \oint_{b_{j}} \omega
$$

We shall now introduce a useful function which is called the Baker-Akhiezer function. We will see that as a consequence of the properties that define it this function satisfies a number of linear partial differential equations. For consistency to obtain, these equations must be solvable simultaneously. Upon imposing this condition on the equations solved by the Baker-Akhiezer function we discover that certain other functions (in terms of which the Baker-Akhiezer function is itself written) automatically satisfy a sequence of non-linear partial differential equations. The first and simplest (i.e., involving the smallest number of independent variables) equation of the sequence is the original Kadomtsev-Petviashvili equation. The full set of equations so described constitute the entire KP sequence. 
Now consider a compact Riemann surface $M$ of genus $g$. Select a general point $\vartheta$ as the origin of a local coordinate system with parameter $z: z(\vartheta)=0$. We specify a polynomial in $z^{-1}$ :

$$
f\left(\frac{1}{z}\right)=\sum_{n=1}^{l} x_{n} z^{-n}
$$

where (for now) $l$ is finite positive, and the $x_{n}$ are real commuting variables. Finally we specify a positive non-special ${ }^{1} \operatorname{divisor} D: D=\left\{P_{1}, \ldots, P_{g}\right\}$ such that $\operatorname{deg} D$ $=\sum_{i=1}^{g} P_{i}=g$. The data set $\mathscr{D}=\left\{M, \vartheta, z, f\left(\frac{1}{z}\right), D\right\}$ has (of course) acquired a name; $\mathscr{D}$ is known to mathematicians as the Krichever map. Having formed the Krichever map we now define the Baker-Akhiezer function as follows. We construct a scalar function $B(z)$ of position on the Riemann surface which is meromorphic everywhere except at the point $\vartheta$, the poles of $B(z)$ occurring precisely at the points of the specified divisor $D=\left\{P_{1}, \ldots, P_{g}\right\}$. In addition the function $B(z)$ is to be constructed such that $B(z) \cdot \exp \left[-f\left(\frac{1}{z}\right)\right]$ is analytic in a neighborhood of $z(\vartheta)=0$. Thus, we require that $B(z)$ possess an essential singularity at $\vartheta$ of the form $B(z) \sim$ const $\exp \left[f\left(\frac{1}{z}\right)\right]$.

We will be able to show that for a non-special divisor $D$ and a general polynomial $f\left(\frac{1}{z}\right)$, the function $B(z)$ is unique up to multiplication by an overall constant. That unique function is the Baker-Akhiezer function

$$
B(z)=\exp \left(\int_{\zeta}^{z} \eta\right) \frac{\theta\left(\boldsymbol{\varphi}_{\zeta}(z)-\boldsymbol{\varphi}_{\zeta}(D)+\mathbf{Y}-\boldsymbol{\kappa}\right)}{\theta\left(\boldsymbol{\varphi}_{\zeta}(z)-\boldsymbol{\varphi}_{\zeta}(D)-\boldsymbol{\kappa}\right)} .
$$

The quantities appearing on the right-hand side are as follows. The symbol $\eta$ denotes a normalized abelian differential form of the second kind:

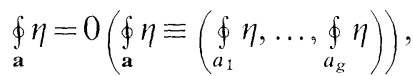

$$
\begin{aligned}
& \oint_{\mathrm{b}} \eta \equiv \mathbf{Y} .
\end{aligned}
$$

$\varphi$ denotes the Abel-Jacobi function defined as

$$
\begin{gathered}
\boldsymbol{\varphi}_{\zeta}(z) \equiv \int_{\zeta}^{z} \omega, \\
\boldsymbol{\varphi}_{\zeta}(D) \equiv \sum_{i=1}^{g} \int_{\zeta}^{P_{i}} \omega ;
\end{gathered}
$$

for the integrals $\int_{\zeta}^{z} \eta$ and $\int_{\zeta}^{z} \omega$ in Eq. (14) the paths of integration connecting $\zeta$ and $z$ are chosen the same. The point $\zeta \neq \vartheta$ is at an arbitrary location on the Riemann

\footnotetext{
${ }^{1}$ A non-special divisor is here basically a divisor such that the associated Abel-Jacobi function is non-singular and invertible
} 
surface, fixed once and for all. Finally, $\boldsymbol{\kappa}$ is the vector of Riemann constants for the world-surface:

$$
\kappa_{i}=\frac{1}{2}\left(1+\Omega_{i i}\right)-\sum_{j \neq i}^{g} \oint_{a_{J}}\left[\omega_{j}(z) \int_{\zeta}^{z} \omega_{i}\right],
$$

i.e., since $\omega_{l}=f_{l}(z) d z$ for a holomorphic function $f_{l}(z)$,

$$
\kappa_{i}=\frac{1}{2}\left(1+\Omega_{i i}\right)-\sum_{j \neq i}^{g} \oint_{a_{j}} f_{j}(z)\left[\int_{\zeta}^{z} f_{i}\left(z^{\prime}\right) d z^{\prime}\right] d z .
$$

Note that the Riemann constants depend trivially on the particular basis chosen for the homology group of the world-surface as well as on the point $\zeta$.

The uniqueness of $B(z)$ may be demonstrated by considering a different path of integration from $\zeta$ to $z$ in which case we have:

$$
\int_{\zeta}^{z} \eta \mapsto \int_{\zeta}^{z} \eta+\oint_{C} \eta
$$

and

$$
\int_{\zeta}^{z} \omega \mapsto \int_{\zeta}^{z} \omega+\oint_{C} \omega
$$

where $C$ is a closed contour. If we decompose the contour $C$ with respect to the chosen homology basis $(\mathbf{a}, \mathbf{b})$ we have

$$
C^{\cdots}=" \sum_{j=1}^{g}\left(m_{j} a_{j}+n_{j} b_{j}\right)
$$

Using the above relation in Eqs. (20) and (21) yields

$$
\begin{aligned}
\int_{\zeta}^{z} \eta \mapsto \int_{\zeta}^{z} \eta+\sum_{j=1}^{g}\left(m_{j} \oint_{a_{3}} \eta+n_{j} \oint_{b_{j}} \eta\right) \\
=\int_{\zeta}^{z} \eta+\sum_{j=1}^{g} n_{j} Y_{j}=\int_{\zeta}^{z} \eta+\mathbf{n} \cdot \mathbf{Y},
\end{aligned}
$$

where we have used Eqs. (15) and (16): also

$$
\int_{\zeta}^{z} \omega_{i} \mapsto \int_{\zeta}^{z} \omega_{i}+m_{i}+\sum_{j=1}^{g} n_{j} \Omega_{i j}
$$

and thus

$$
\int_{\zeta}^{z} \omega \mapsto \int_{\zeta}^{z} \omega+\mathbf{m}+\boldsymbol{\Xi}
$$

where as before

$$
\Xi \equiv \sum_{j=1}^{g} n_{j} \oint_{b_{j}} \omega
$$

If we recall that Riemann's theta-function has the following property:

$$
\theta(\mathbf{z}+\mathbf{m}+\boldsymbol{\Xi})=\exp \left[-\frac{\pi i}{2} \mathbf{n} \cdot(\boldsymbol{\Xi}+2 \mathbf{z})\right] \theta(\mathbf{z}),
$$


and employ Eqs. (23) and (25) in Eq. (14), we find that $B(z)$ is indeed invariant under a change of integration path from $\zeta$ to $z$ :

$$
\begin{aligned}
B(z)= & \exp \left(\int_{\zeta}^{z} \eta\right) \frac{\theta\left(\boldsymbol{\varphi}_{(}(z)-\boldsymbol{\varphi}(D)+\mathbf{Y}-\mathbf{\kappa}\right)}{\theta\left(\boldsymbol{\varphi}_{(}(z)-\boldsymbol{\varphi}_{(}(D)-\boldsymbol{\kappa}\right)} \\
& \rightarrow \exp \left(\int_{\zeta}^{z} \eta+\mathbf{n} \cdot \mathbf{Y}\right) \cdot \frac{\exp \left[-\frac{1}{2} \mathbf{n} \cdot \mathbf{\Xi}-\mathbf{n} \cdot\left(\boldsymbol{\varphi}_{(}(z)-\boldsymbol{\varphi}_{\zeta}(D)+\mathbf{Y}-\boldsymbol{\kappa}\right)\right]}{\exp \left[-\frac{1}{2} \mathbf{n} \cdot \mathbf{\Xi}-\mathbf{n} \cdot\left(\boldsymbol{\varphi}_{z}(z)-\boldsymbol{\varphi}_{\zeta}(D)-\boldsymbol{\kappa}\right)\right]} \\
& \times \frac{\theta\left(\boldsymbol{\varphi}_{\zeta}(z)-\boldsymbol{\varphi}_{\zeta}(D)+\mathbf{Y}-\mathbf{\kappa}\right)}{\theta\left(\boldsymbol{\varphi}_{\zeta}(z)-\boldsymbol{\varphi}_{\zeta}(D)-\boldsymbol{\kappa}\right)}=B(z)
\end{aligned}
$$

Now if we choose for the abelian differential $\eta(z)=d f\left(z^{-1}\right)+O(d z)$, where $f\left(z^{-1}\right)$ is given in Eq. (13), then

$$
\int \eta=f+(\text { regular terms }) \text {. }
$$
With this choice, $B(z)$ as defined in Eq. (14) satisfies $B(z) \sim \operatorname{const} \cdot \exp \left[f\left(\frac{1}{z}\right)\right]$ in a
neighborhood of $\vartheta$ and all the necessary criteria are met.

Given the Baker-Akhiezer function we now deduce the linear equations it satisfies and then infer the sequence of KP equations. To do this we will eventually let the upper limit of summation $/$ in Eq. (13) tend to infinity, but for simplicity we first set $l=3$. Thus we have

$$
f\left(z^{-1}\right)=x_{1} z^{-1}+x_{2} z^{-2}+x_{3} z^{-3} .
$$

If we now employ Eq. (20) in Eq. (14) we may express the Baker-Akhiezer function as the infinite series

$$
B(z)=e^{x_{1} z^{-1}+x_{2} z^{-2}+x_{3} z^{-3}}\left[g_{0}(\vec{x})+z g_{1}(\vec{x})+z^{2} g_{2}(\vec{x})+\ldots\right] \equiv B(\vec{x}, z) .
$$

Here $\vec{x} \equiv\left(x_{1}, x_{2}, x_{3}\right)$ and the $g_{i}(i=0,1, \ldots)$ are calculated subject to the normalization condition $g_{0}(\vec{x}) \equiv 1$. If we now define

$$
\psi(\vec{x}) \equiv-2 \frac{\partial}{\partial x_{1}} g_{1}(\vec{x})
$$

and

$$
\frac{1}{3} \xi(\vec{x}) \equiv\left[\frac{\partial^{2}}{\partial x_{1}^{2}}+g_{1}(\vec{x}) \frac{\partial}{\partial x_{1}}\right] g_{1}(\vec{x})-\frac{\partial}{\partial x_{1}} g_{2}(\vec{x}),
$$

it is straightforward to verify that the right-hand side of Eq. (31) satisfies the following two equations:

$$
\begin{gathered}
{\left[\frac{\partial^{2}}{\partial x_{1}^{2}}-\frac{\partial}{\partial x_{2}}+\psi(\vec{x})\right] B(z)=\exp \left[f\left(\frac{1}{z}\right)\right] \cdot O(z)} \\
{\left[\frac{\partial^{3}}{\partial x_{1}^{3}}+\frac{3}{2} \psi(\vec{x}) \frac{\partial}{\partial x_{1}}-\frac{\partial}{\partial x_{3}}+\xi(\vec{x})\right] B(z)=\exp \left[f\left(\frac{1}{z}\right)\right] \cdot O(z) .}
\end{gathered}
$$

Upon defining the ordinary differential operators

$$
\hat{H}_{2} \equiv \frac{\partial^{2}}{\partial x_{1}^{2}}+\psi(\vec{x}) \text {, }
$$


and

$$
\hat{H}_{3} \equiv \frac{\partial^{3}}{\partial x_{1}^{3}}+\frac{3}{2} \psi(\vec{x}) \frac{\partial}{\partial x_{1}}+\xi(\vec{x})
$$

(the motivation for the subscripts will appear in the sequel) we discover that the Baker-Akhiezer function [with $f\left(z^{-1}\right)$ as in Eq. (30)] satisfies the following two differential equations known as the Lax pair:

$$
\begin{aligned}
& \frac{\partial B}{\partial x_{2}}=\hat{H}_{2} B, \\
& \frac{\partial B}{\partial x_{3}}=\hat{H}_{3} B .
\end{aligned}
$$

To prove that Eqs. (36) and (37) may be consistently solved we first note that $\widetilde{B}_{2}$ $\equiv\left(\hat{H}_{2}-\frac{\partial}{\partial x_{2}}\right) B$ and $\widetilde{B}_{3} \equiv\left(\hat{H}_{3}-\frac{\partial}{\partial x_{3}}\right) B$ are both Baker-Akhiezer functions since they satisfy the defining criteria. However, from Eq. (34) we find that $\lim _{z \rightarrow 0}\left\{\widetilde{B}_{2} \cdot \exp \left[-f\left(\frac{1}{z}\right)\right]\right\}$ and $\lim _{z \rightarrow 0}\left\{\widetilde{B}_{3} \cdot \exp \left[-f\left(\frac{1}{z}\right)\right]\right\}$ both vanish. Since Baker-Akhiezer functions are unique, we therefore have $\widetilde{B}_{2}=\widetilde{B}_{3} \equiv 0$ everywhere on the world-surface $M$.

In view of this result we impose the consistency of Eqs. (36) and (37) by demanding that the commutator of $\widetilde{B}_{2}$ and $\widetilde{B}_{3}$ vanishes identically

$$
\left[\hat{H}_{2}-\frac{\partial}{\partial x_{2}}, \hat{H}_{3}-\frac{\partial}{\partial x_{3}}\right]=0 \text {. }
$$

If we explicitly calculate this commutator and invoke the uniqueness of the BakerAkhiezer function, we derive the following partial differential equations:

$$
\begin{gathered}
\frac{\partial \psi(\vec{x})}{\partial x_{2}}+\frac{\partial^{2} \psi(\vec{x})}{\partial x_{1}^{2}}-\frac{4}{3} \frac{\partial \xi(\vec{x})}{\partial x_{1}}=0 ; \\
\frac{\partial \psi(\vec{x})}{\partial x_{3}}-\frac{\partial \xi(\vec{x})}{\partial x_{2}}-\frac{\partial^{3} \psi(\vec{x})}{\partial x_{1}^{3}}-\frac{3}{2} \psi(\vec{x}) \frac{\partial \psi(\vec{x})}{\partial x_{1}}+\frac{\partial^{2} \xi(\vec{x})}{\partial x_{1}^{2}}=0 .
\end{gathered}
$$

Upon eliminating $\xi(\vec{x})$ from Eqs. (39) we obtain the first equation of the KP sequence, the original Kadomtsev-Petviashvili equation in three real variables:

$$
\frac{\partial^{4} \psi(\vec{x})}{\partial x_{1}^{4}}+6 \psi(\vec{x}) \frac{\partial^{2} \psi(\vec{x})}{\partial x_{1}^{2}}+6\left[\frac{\partial \psi(\vec{x})}{\partial x_{1}}\right]^{2}+3 \frac{\partial^{2} \psi(\vec{x})}{\partial x_{2}^{2}}-4 \frac{\partial^{2} \psi(\vec{x})}{\partial x_{1} \partial x_{3}}=0 .
$$

In fact (as mentioned in the introduction), this non-linear fourth-order partial differential equation (actually a very slightly different equation) was first constructed by Kadomtsev and Petviashvili to study the propagation of long, shallow waves in a plasma.

To generate the full sequence of KP equations we now abandon Eq. (30) and let the upper limit of summation in Eq. (13) tend to infinity, yielding

$$
f\left(\frac{1}{z}\right)=\sum_{n=1}^{\infty} x_{n} z^{-n}
$$


so we now have

$$
\vec{x}=\left(x_{1}, \ldots\right),
$$

and the series expansion of the Baker-Akhiezer function in Eq. (31) depends on an infinite number of real variables. We now generalize the Lax pair of equations [Eqs. (36) and (37)] by requiring $B(\vec{x}, z)$ to satisfy

$$
\frac{\partial}{\partial x_{n}} B(\vec{x}, z)=\hat{H}_{n}\left(\vec{x}, \frac{\partial}{\partial x_{1}}\right) B(\vec{x}, z),
$$

where

$$
\hat{H}_{n}\left(\vec{x}, \frac{\partial}{\partial x_{1}}\right) \equiv \frac{\partial^{n}}{\partial x_{1}^{n}}+\sum_{m=2}^{n} \psi_{n, n-m}(\vec{x}) \frac{\partial^{n-m}}{\partial x_{1}^{n-m}},
$$

where $n \geqq 2$. For example, with $n=2,3$ we find

$$
\left\{\begin{array}{l}
\hat{H}_{2}=\frac{\partial^{2}}{\partial x_{1}^{2}}+\psi_{2,0}(\vec{x}) \\
\hat{H}_{3}=\frac{\partial^{3}}{\partial x_{1}^{3}}+\psi_{3.1}(\vec{x}) \frac{\partial}{\partial x_{1}}+\psi_{3.0}(\vec{x}),
\end{array}\right.
$$

so that we recover the Lax pair, and hence the original KP equation, if we make the identification $\psi_{2,0}(\vec{x}) \equiv \psi(\vec{x}), \psi_{3,1}(\vec{x}) \equiv \frac{3}{2} \psi(\vec{x})$, and $\psi_{3,0}(\vec{x}) \equiv \xi(\vec{x})$. To observe the full $\mathrm{KP}$ sequence we differentiate the $n^{\text {th }}$ equation in Eq. (43) with respect to $x_{m}$, and we differentiate the $m^{\text {th }}$ equation with respect to $x_{n}$ and subtract the results, which yields

$$
\left\{\frac{\partial}{\partial x_{n}} \hat{H}_{m}\left(\vec{x}, \frac{\partial}{\partial x_{1}}\right)-\frac{\partial}{\partial x_{m}} \hat{H}_{n}\left(\vec{x}, \frac{\partial}{\partial x_{1}}\right)-\left[\hat{H}_{n}, \hat{H}_{m}\right]\right\} B(\vec{x}, z)=0 .
$$

The above equation is shorthand for the entire KP sequence of equations, since it embodies infinitely many constraints on the coefficient functions $\psi_{j, k}$. In principle after an infinite number of integrations all the $\psi_{j, k}$ are determined in terms of a particular one; these relations are in the form of infinitely many non-linear partial differential equations with respect to an infinite number of independent variables.

It has been asserted that the KP equations can be solved exactly. We will show presently how this goes by deducing the solution for the original KP equation (more precisely, we will show how one obtains the so-called quasi-periodic solutions). However, before doing so we will first take a brief detour by writing the $\mathrm{KP}$ equations in a different form to bring infinite-dimensional Lie algebras into the discussion.

A striking feature of the KP equations is the natural emergence of the Virasoro algebra from the solution set. The way this comes about is essentially as follows. It is usually the case that a system of exactly solvable non-linear partial differential equations has associated with it a group of transformations of the solutions of the equations. Such transformations comprise a Bäcklund group. The generic element of the Bäcklund group takes a given solution of an equation in the "integrable" system into another solution. The totality of the elements transform all of the solutions into one another. 
In the case of the KP sequence the Bäcklund group is in fact an infinitedimensional affine Lie group. Furthermore, the infinitesimal generators of the group satisfy the Virasoro algebra with central extension.

We would now like to see how the solutions of the KP sequence are transformed into one another by the Virasoro algebra, and as well, to see the infinite set of solutions from a clear vantage point. To this end it is very useful to rewrite the KP equations using a clever change of variables suggested by Hirota [29]. First we shall introduce the Hirota bilinear operator, $\hat{P}$, as follows. Associated with a differential operator in one real variable, $D(x)$, we define the Hirota operator acting on the product of two functions of $x$ :

$$
\left.\hat{P}(D(x))[f(x) \cdot g(x)] \equiv D(y)[f(x+y) \cdot g(x-y)]\right|_{y=0} .
$$

For example

$$
\hat{p}\left(\frac{\partial}{\partial x}\right)[f(x) \cdot g(x)]=f^{\prime} g-f g^{\prime},
$$

and

$$
\hat{P}\left(\frac{\partial^{2}}{\partial x^{2}}\right)[f(x) \cdot g(x)]=f g^{\prime \prime}-2 f^{\prime} g^{\prime}+f^{\prime \prime} g .
$$

For later convenience we list here the following results:

$$
\begin{aligned}
\hat{P}(\partial)(f \cdot f) & =0, \\
\hat{P}\left(\partial^{2}\right)(f \cdot f) & =2\left(f f^{\prime \prime}-f^{\prime 2}\right), \\
\hat{P}\left(\partial^{3}\right)(f \cdot f) & =0, \\
\hat{P}\left(\partial^{4}\right)(f \cdot f) & =2\left(f f^{\prime \prime \prime}-4 f^{\prime} f^{\prime \prime \prime}+3 f^{\prime \prime 2}\right),
\end{aligned}
$$

etc. The Hirota bilinear operator associated with differential operators in several real variables is a simple generalization of Eq. (47):

$$
\begin{aligned}
& \hat{P}\left(D\left(x_{1}\right), D\left(x_{2}\right), \ldots\right)[f(\vec{x}) \cdot g(\vec{x})] \\
& \left.\equiv \hat{P}\left(\partial_{x 1}, \partial_{x 2}, \ldots\right)\left[f\left(x_{1}+x_{1}^{\prime}, x_{2}+x_{2}^{\prime}, \ldots\right) g\left(x_{1}-x_{1}^{\prime}, x_{2}-x_{2}^{\prime}, \ldots\right)\right]\right|_{x_{1}^{\prime}=x_{2}^{\prime}=\ldots=0} .
\end{aligned}
$$

Having introduced Hirota's bilinear operator we now define Hirota's tau function $\tau(\vec{x})$ through the following change of variables:

$$
\psi(\vec{x})=2 \frac{\partial^{2}}{\partial x_{1}^{2}} \log \tau(\vec{x})
$$

where $\psi(\vec{x})$ appeared first in Eq. (32). Substituting Eq. (55) in Eq. (40) and using the definition of the bilinear operator we obtain, after some algebra, the bilinear form of the original KP equation,

$$
\hat{P}\left(D_{1}^{4}+3 D_{2}^{2}-4 D_{1} D_{3}\right) \tau \cdot \tau=0 .
$$

To now generate the entire KP sequence we define the Schur polynomials [29] $S_{n}(\vec{x})(n=0,1, \ldots)$ via

$$
\exp \left(\sum_{n=1}^{\infty} x_{n} z^{-n}\right)=\sum_{n=0}^{\infty} z^{-n} S_{n}(\vec{x}),
$$


so that

$$
\left\{\begin{array}{l}
S_{0}(\vec{x})=1 \\
S_{1}(\vec{x})=x_{1} \\
S_{2}(\vec{x})=x_{2}+\frac{1}{2} x_{1}^{2} \\
S_{3}(\vec{x})=x_{3}+x_{2} x_{1}+\frac{1}{3} x_{1}^{3}, \text { etc. }
\end{array}\right.
$$

We will also need to introduce the vector

$$
\vec{p} \equiv\left(\frac{\partial}{\partial x_{1}}, \frac{1}{2} \frac{\partial}{\partial x_{2}}, \frac{1}{3} \frac{\partial}{\partial x_{3}}, \ldots\right)
$$

With these definitions it is possible to show that the bilinear equation $\left(\vec{\eta}=\left(\eta_{1}, \eta_{2}, \ldots\right)\right.$ are arbitrary $\left.(\neq 0)\right)$

$$
\hat{P}\left[\sum_{l=0}^{\infty} S_{l}(-2 \vec{\eta}) S_{l+1}(\vec{p}) \exp \left(\sum_{j=1}^{\infty} \eta_{j} \frac{\partial}{\partial x_{j}}\right)\right] \tau(\vec{x}) \cdot \tau(\vec{x})=0
$$

is [in similarity with Eq. (46)] shorthand for the entire KP sequence. This can be seen (with some effort) by equating to zero each coefficient of monomials in the $\eta_{j}$. In this way one in principle may recover the infinite set of non-linear partial differential equations.

Now to see the action of the Virasoro algebra we recall Eqs. (47) and (54), we observe that (here $c$ is a constant)

$$
\hat{P}(D(x))[f(x+c) \cdot g(x+c)]=0
$$

and

$$
\hat{P}(D(x))\left[e^{c x} f(x) \cdot e^{c x} g(x)\right]=0,
$$

provided $\hat{P}(D(x))[f(x) \cdot g(x)]=0$. With this in mind, following Lepowsky and Wilson [31], we introduce the operator

$$
\widehat{V}(z, \zeta) \equiv \exp \left(\sum_{n=1}^{\infty} x_{n} z^{-n}-\sum_{n=1}^{\infty} x_{n} \zeta^{-n}\right) \exp \left(-\sum_{n=1}^{\infty} p_{n} z^{n}+\sum_{n=1}^{\infty} p_{n} \zeta^{n}\right),
$$

where the $p_{n}$ are defined in Eq. (59). If we now denote the so-called $N$-soliton solution $^{2}$ of the KP sequence by $\tau_{N}\left(\right.$ actually the solution is $\left.2 \frac{\partial^{2}}{\partial x_{1}^{2}} \log \tau_{N}\right)$ with

$$
\tau_{N}=\exp \left[\sum_{l=1}^{N} \alpha_{l} \hat{V}(z, \zeta)\right] \cdot 1
$$

(the $\alpha_{l}$ form a sequence of complex numbers) it can be verified that

$$
e^{\alpha \hat{V}} \tau_{N}=\tau_{N+1},
$$

where $\alpha \notin\left\{\alpha_{l}\right\}$. Thus $\hat{V}$ acts on the solutions of the KP equation essentially as an infinitesimal Bäcklund transformation. Since 1 and the operators $\hat{V}$ span a Lie algebra [31], the group orbit of the highest weight (vacuum) vector 1 is isomorphic to the solution space.

\footnotetext{
${ }^{2}$ This describes a "wave-packet" formed from $N$ separate waves
} 
After this digression to make contact with the Virasoro algebra we will now deduce the form of the quasi-periodic solutions of the KP equation. These solutions are so named because of the appearance in them of the (quasi-periodic) Riemann theta-functions.

We consider again the original Kadomtsev-Petviashvili equation [Eq. (40)] in three real independent variables. The solution $\psi(\vec{x})$ for this equation is given in Eq. (32) in terms of an expansion coefficient for the Baker-Akhiezer function which appears in Eq. (31). We utilize both Eq. (31) and Eq. (14) to write the relevant Baker-Akhiezer function as

$$
\begin{aligned}
B(z)= & \exp \left(x_{1} \int_{\zeta}^{z} \eta+x_{2} \int_{\zeta}^{z} v+x_{3} \int_{\zeta}^{z} \lambda\right) \\
& \times \frac{\theta\left(\boldsymbol{\varphi}_{\zeta}(z)-\boldsymbol{\varphi}_{\zeta}(D)+x_{1} \mathbf{Y}+x_{2} \mathbf{N}+x_{3} \mathbf{\Lambda}-\boldsymbol{\kappa}\right.}{\theta\left(\boldsymbol{\varphi}_{\zeta}(z)-\boldsymbol{\varphi}_{\zeta}(D)-\boldsymbol{\kappa}\right)},
\end{aligned}
$$

where here

$$
\begin{aligned}
& \eta=d\left(z^{-1}\right)+d(O(z)), \\
& v=d\left(z^{-2}\right)+d(O(z)), \\
& \lambda=d\left(z^{-3}\right)+d(O(z)),
\end{aligned}
$$

and

$$
\begin{aligned}
& \mathbf{Y}=\oint_{\mathbf{b}} \eta, \\
& \mathbf{N}=\oint_{\mathbf{b}} v,
\end{aligned}
$$

and

$$
\mathbf{\Lambda}=\oint_{\mathbf{b}} \lambda .
$$

Using Eq. (31) we now write the logarithm of the Baker-Akhiezer function near the point $\vartheta$ of the Krichever map as

$$
\log B(z)=z^{-3} x_{3}+z^{-2} x_{2}+z^{-1} x_{1}+g_{0}(\vec{x})+z\left[g_{1}(\vec{x})+\alpha x_{1}+\beta x_{2}+\gamma x_{3}\right]+O\left(z^{2}\right),
$$

where $\alpha, \beta, \gamma$ are constants. Comparing this with Eq. (66) we see that in a neighborhood of $\vartheta$ the coefficient of order $z$ of

$$
\log \left[\frac{\theta\left(\boldsymbol{\varphi}_{\zeta}-\boldsymbol{\varphi}_{\zeta}(D)+x_{1} \mathbf{Y}+x_{2} \mathbf{N}+x_{3} \Lambda-\boldsymbol{\kappa}\right)}{\theta\left(\boldsymbol{\varphi}_{\zeta}(z)-\boldsymbol{\varphi}_{\zeta}(D)-\boldsymbol{\kappa}\right)}\right]
$$

is

$$
g_{1}(\vec{x})+\alpha x_{1}+\beta x_{2}+\gamma x_{3} .
$$

Now we employ the expansion

$$
\boldsymbol{\varphi}_{\zeta}(z)=\boldsymbol{\varphi}_{\zeta}(\boldsymbol{\vartheta})-z \mathbf{Y}+O\left(z^{2}\right)
$$

which after choosing $\zeta \rightarrow \vartheta$ becomes

$$
\boldsymbol{\varphi}_{\zeta}(z)=-z \mathbf{Y}+O\left(z^{2}\right),
$$


to deduce

$$
g_{1}(\vec{x})+\alpha x_{1}+\beta x_{2}+\gamma x_{3}=-\frac{\partial}{\partial x_{1}} \log \theta\left(x_{1} \mathbf{Y}+x_{2} \mathbf{N}+x_{3} \Lambda-\boldsymbol{\varphi}_{\zeta}(D)-\boldsymbol{\kappa}\right)+\text { const } .
$$

When we combine Eq. (78) with Eq. (32) we discover that the quasi-periodic solution of the Kadomtsev-Petviashvili equation is

$$
\psi(\vec{x})=-2 \frac{\partial}{\partial x_{1}} g_{1}(\vec{x})=2 \frac{\partial^{2}}{\partial x_{1}^{2}} \log \theta\left(x_{1} \mathbf{Y}+x_{2} \mathbf{N}+x_{3} \Lambda-\boldsymbol{\varphi}_{\zeta}(D)-\mathbf{\kappa}\right)+\text { const } .
$$

Note that the solution depends on the chosen non-special divisor $D$, and hence on a point in the abelian variety associated with the lattice defining the theta-function in Eq. (79). In fact, it can be shown that the solutions determine a straight line on the Jacobi variety of the Riemann surface associated with $\theta$.

Here we have considered the original KP equation for simplicity, but analogous solutions can be found for the entire KP sequence by extending the above analysis. We choose to employ Eq. (55) to work with the tau-function rather than the solution $\psi(\vec{x})$ itself.

Following Fay [32], we write the tau-function for the KP sequence [here now $\left.\vec{x}=\left(x_{1}, x_{2}, \ldots\right)\right]$ as

$$
\tau(\vec{x})=e^{\frac{1}{2} Q(\vec{x})} \theta(\vec{x} \cdot \boldsymbol{\eta}+\xi)
$$

where

$$
\begin{gathered}
Q(\vec{x})=\sum_{m \cdot n=1}^{\infty} \sigma_{m n} x_{m} x_{n}, \\
\sigma_{m n}=\left.\frac{-1}{(m-1) !(n-1) !} \frac{\partial^{m+n}}{\partial u^{m} \partial v^{n}} \log \frac{E(u, v)}{u-v}\right|_{u=v=0},
\end{gathered}
$$

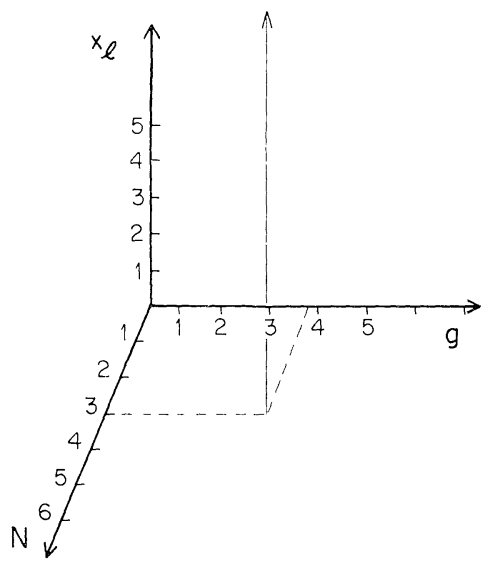

Fig. 2. This figure depicts the "partial parameter space" for the quasi-periodic solutions of the KP equations with a degenerating solution locus 
with $u, v \in \mathbb{C}$ and $E(u, v)$ the "prime-form" for the Riemann surface. In the argument for the theta-function, $\xi$ is arbitrary (parametrizing position on the Jacobi variety), and

$$
\vec{x} \cdot \boldsymbol{\eta}=-\sum_{n=1}^{\infty} n x_{n} \oint_{\mathbf{b}} z^{-n} \frac{d z}{z}
$$

(of course $\theta(\mathbf{z})=\theta\left[\begin{array}{l}0 \\ 0\end{array}\right](\mathbf{z} \mid \Omega)$ as in Eq. (11)).

With the tau-function given as above, we can give a partial parametrization (ignoring, for instance, position on the Jacobi variety) of quasi-periodic solutions by constructing the space indicated in Fig. 2. There are three axes, for genus, number of independent variables, and soliton number respectively. Here it should be noted that motion along the genus and soliton number axes is generated by the Bäcklund transformations discussed previously [33].

\section{Outlook}

The path integral with respect to the string coordinates for the bosonic string with first-quantized action

$$
S=\frac{i}{4 \pi} \int X \partial \bar{\partial} X
$$

has been evaluated by Vafa [26]. Here the functional integral is evaluated for a string producing a world-surface with the topology of a sphere with an arbitrary finite number of handles and with exactly one hold cut out of the surface. This path integral defines a functionai on the space of values of the two-dimensional quantum fields at the boundary of the world-surface. Following Vafa, we will denote such a functional by $\langle x \mid \phi\rangle$, and this symbol will specify a state in the Hilbert space referring to the boundary.

We now introduce the following isomorphism between the first-quantized string oscillators and a discrete, infinite set of commuting real variables [30] (one each for the left-moving modes and the right-moving modes):

$$
\left.\begin{array}{l}
a_{n}^{L(R)} \leftrightarrow \frac{1}{i \sqrt{n}} \frac{\partial}{\partial x_{n, L(R)}} \\
a_{n}^{\dagger L(R)} \leftrightarrow i \sqrt{n} x_{n, L(R)}
\end{array}\right\} \quad(n=1, \ldots),
$$

where the algebra satisfied by the string oscillators is

$$
\left[a_{n}^{L}, a_{n}^{\dagger L}\right]=\left[a_{n}^{R}, a_{n}^{\dagger R}\right]=1 \text {. }
$$

Using the isomorphism in Eq. (85) Vafa has established the following relation between the states in the Hilbert space and the quasi-periodic solutions of the KP equations:

$$
\langle x \mid \phi\rangle=\left[c_{L} \tau\left(\vec{x}_{L}\right)\right] \cdot\left[c_{R} \tau\left(\vec{x}_{R}\right)\right],
$$

where the $c_{\tilde{L}, R}$ are (undetermined) complex constants. Recalling Eq. (55) we write now

$$
\psi\left(\vec{x}_{L, R}\right)=2 \frac{\partial^{2}}{\partial x_{1_{L, R}}^{2}} \log \tau\left(\vec{x}_{L, R}\right) \equiv \psi_{L, R}
$$


and define

$$
2 \log \langle x \mid \phi\rangle \equiv\langle x \mid \psi\rangle \text {. }
$$

Then we have that the sum of the second derivatives for the left-and right-movers, taken with respect to the ground states, of twice the logarithm of the string state-vector, is equal to the sum of the solutions of two independent KP sequences:

$$
\left(\frac{\partial^{2}}{\partial x_{1, L}^{2}}+\frac{\partial^{2}}{\partial x_{1, R}^{2}}\right)\langle x \mid \psi\rangle=\psi_{L}+\psi_{R} .
$$

From the analysis of Vafa we know that the same description can be obtained starting from a Lagrangian describing a fermionic theory rather than the bosonic Lagrangian given in Eq. (84). However, the discussion throughout has been applied to free two-dimensional field theory. Should one decide to adopt the Green-Schwarz picture instead of the Neveu-Ramond-Schwarz picture it is not clear how to proceed. Since these two first-quantized pictures are often held to describe the same theory, it should be interesting to extend the KP equations so as to account for the Green-Schwarz picture.

It should be noted that the lower elements of the KP sequence, which depend on a finite number of independent variables, may be used to furnish what could be called a low-energy approximation to the string, since then only a subset of all the string modes are included in the description.

Finally, it may be useful to investigate extensions of the KP equations which augment the real, commuting (even) independent variables with an odd set. Such supersymmetric extensions of the KP sequence have been formally defined [34]; the immediate problem to solve for application to a fundamental formulation of string theory is the determination of explicit algebro-geometric solutions, for surfaces of finite and infinite genus. Work on all these topics is actively in progress.

Acknowledgements. I wish to acknowledge useful discussions with M. Douglas and R. Rohm.

\section{References}

1. Belavin, A.A., Polyakov, A.M., Zamolodchikov, A.B.: Nucl. Phys. B 241, 333 (1984)

2. Friedan, D.: Nucl. Phys. B 271, 540 (1986)

3. Hata, H., Itoh, K., Kugo, T., Kunitomo, H., Ogawa, K.: Covariant string field theory. Kyoto University preprint RIFP-660 (1986)

4. Horowitz, G., Lykken, J., Rohm, R., Strominger, A.: Phys. Rev. Lett. 57, 283 (1986)

5. Neveu, A., West, P.C.: Nucl. Phys. B 268, 125 (1986)

6. Witten, E.: Nucl. Phys. B 268, 253 (1986)

7. Polyakov, A.M.: Phys. Lett. 103 B, 207 (1981)

8. Alvarez, O.: Nucl. Phys. 216, 125 (1983)

9. Moore, G., Nelson, P.: Nucl. Phys. B 266, 58 (1986)

10. D'Hoker, E., Phong, D.: Nucl. Phys. B 269, 205 (1986)

11. Gilbert, G.: Nucl. Phys. B 277, 102 (1986)

12. Belavin, A.A., Knizhnik, V.: Phys. Lett. 168 B, 201 (1986)

13. Manin, Yu.: Phys. Lett. 172 B, 184 (1986)

14. Moore, G.: Phys. Lett. 176 B, 389 (1986)

15. Gava, E., Iengo, R., Jayoraman, T., Ramachandran, R.: Phys. Lett. 168 B, 207 (1986)

16. Alvarez-Gaumé, L., Gomez, C.: Phys. Lett. 190 B, 55 (1987) 
17. Friedan, D., Shenker, S.: Phys. Lett. 175 B, 287 (1986)

18. Manin, Yu.: Funkt. Anal. 20(3), 88 (1986)

19. Segal, G., Wilson, G.: Pub. IHES 61, 1 (1985)

20. Witten, E.: Quantum field theory, Grassmannians, and algebraic curves. Commun. Math. Phys. 113, 529 (1988)

21. Shiota, T.: Inv. Math. 83, 333 (1986)

22. Mulase, M.: J. Differ. Geom. 19, 403 (1984)

23. Dubrovin, B., Krichever, I.M.: Sov. Sci. Rev. 3, 1 (1982)

24. Kadomtsev, B., Petviashvili, V.: Dokl. An. SSR. 192(4), 753 (1970)

25. Date, E., Jimbo, M., Kashiwara, M., Miwa, T.: J. Phys. Soc. Jpn. 50, 3806 (1981)

26. Vafa, C.: Phys. Lett. 190 B, 47 (1987)

27. Moore, G.: Atkin-Lehner Symmetry. Harvard University preprint HUTP-87/A013 (1987)

28. McKean, H.P., Trubowitz, E.: Bull. Am. Math. Soc. 84, 1042 (1978)

29. Hirota, R.: In: Lecture Notes in Mathematics, Vol. 515. Berlin, Heidelberg, New York: Springer 1976

30. Kac, V.: Infinite dimensional Lie algebras. Cambridge: Cambridge University Press 1985

31. Lepowsky, J., Wilson, R.: Commun. Math. Phys. 62, 43 (1978)

32. Fay, J.: Bilinear identities. Haverford College preprint

33. Ishibashi, N., Matsuo, Y., Ooguri, H.: Soliton equations and free fermions on Riemann surfaces. University of Tokyo preprint UT-499 $(1986)$

34. Manin, Yu., Radul, A.: Commun. Math. Phys. 98, 65 (1985)

Communicated by S.-T. Yau

Received January 28, 1988 